\title{
Antiviral Efficacy of an Aquatic Disinfectant Tablet Composed to Calcium Hypochlorite Against Red Sea Bream Iridovirus
}

\author{
Chun-Nam Cha', Yeo-Eun Lee ${ }^{2}$, In-Jin Kang ${ }^{2}$, Chang-Yeul Yoo ${ }^{3}$, Eun-Kee Park', Suk Kim, and Hu-Jang Lee* \\ Research Institute of Live Sciences, College of Veterinary Medicine, Gyeongsang National University, \\ Chinju 600-701, Korea \\ ${ }^{1}$ Engineering Research Institute, Department of Industrial Systems Engineering, \\ Gyeongsang National University, Chinju 600-701, Korea \\ ${ }^{2}$ Department of Environmental Health, Graduate School of Public Health, \\ Gyeongsang National University, Chinju 660-751, Korea \\ ${ }^{3}$ Department of Computer Information, Gyeongnam Provincial Namhae College, Namhae 668-801, Korea \\ ${ }^{4}$ Department of Medical Humanities and Social Medicine, College of Medicine, Kosin University, Busan 602-703, Korea \\ (Received June 17, 2012/Revised June 29, 2012/Accepted July 8, 2012)
}

\begin{abstract}
In this study, the veridical efficacy of an aquatic disinfectant tablet composed to calcium hypochlorite against red sea bream iridovirus (RBIV). A veridical efficacy was determined with the viability of RBIV contacted with the disinfectant in viral stock cultured in fat head minnow cell line. An aquatic disinfectant tablet and RBIV were reacted on the distilled water (DW), hard water (HW) or organic matter suspension (OM) condition. On DW and HW condition, RBIV was inactivated with 25,000 fold dilutions of an aquatic disinfectant tablet. With the investigation of the antiviral effect of the disinfectant on OM condition, RBIV was inactivated on 22,000 fold dilutions of an aquatic disinfectant tablet. As an aquatic disinfectant tablet possesses veridical efficacy against RBIV, the disinfectant solution can be used to limit the spread of cultured marine fish viral disease.
\end{abstract}

Key words: Aquatic disinfectant tablet, Red sea bream iridovirus, Calcium hypochlorite, Disinfectant efficacy

\section{Introduction}

Iridoviruses have been implicated as the cause of severe disease, mortality and economic loss in farmed food fish and ornamental fish, as well as in wild fish ${ }^{1)}$. Iridoviruses are icosahedral cytoplasm DNA viruses that have been isolated from invertebrate and vertebrate host species. Iridovirus has a large double-stranded DNA genome and a size of 120-300 $\mathrm{nm}$ in diameter ${ }^{2}$. Red sea bream iridovirus (RSIV) is a piscine iridovirus and causes an acute and highly contagious disease, designated as red sea bream iridoviral disease $(\text { RSIVD })^{3)}$. The number of reports of iridovirus infection of fish has increased rapidly over the past decade, with abundant reports from China, Japan, Korea, and Taiwan ${ }^{4)}$. Since 1990, outbreaks of RSIVD have resulted in high mortality in cultured red sea bream in the southwestern part of Japan, primarily in the summertime $e^{5)}$.

In Korea, many outbreak cases by RSIV have been

\footnotetext{
*Correspondence to: Hu-Jang Lee, DVM, MPH, PhD, College of Veterinary Medicine, Gyeongsang National University, 900 Gajwadong, Chinju 660-701, Korea

Tel: 82-55-772-2352, Fax: 82-55-772-2308

E-mail: hujang@gnu.ac.kr
}

reported in different aquatic farms beginning in 1998. In 2003, iridoviral epizootics occurred in flounders cultured in the southern part of Korea, and 13 iridoviruses were isolated from cultured flounders ${ }^{6}$. Fishes infected by RSIV have severe anemia and show petechia of the gills, congestion of the liver, and hypertrophy of the spleen and kidney ${ }^{7)}$.

Water supplies for seed production and aquaculture often provide an efficient means for the introduction and spread of infectious diseases. A pathogen-free water source is essential for success in aquaculture. Surface waters commonly used in aquaculture come from coastal waters or rivers and may contain some fish pathogens and such open water supplies should not be used without treatment. Disinfection of wastewater before discharging is necessary to avoid the pathogen contamination in the environment ${ }^{8)}$.

Kasai et $a l^{8)}$ reported that ultraviolet radiation was killed iridovirus and hypochlorite produced by electrolysis showed viricidal effects. Due to their resistance to drying and disinfection, epizootic hematopoietic necrosis (EHN), a systemic iridoviral disease of fish, is presumed to persist for months or years on infected farms in the water, pond sediments, plants and equipment ${ }^{9}$.

Many disinfectants such as iodophore, sodium hypochlorite solution, benzalconium chloride solution, saponated cresole 
solution, formaldehyde solution, and potassium pennanganate solution, were reported to have a virucidal activity against various fish pathogenic viruses ${ }^{10)}$.

However, there is not the efficacy test for the disinfectant composed of calcium hypochlorite against iridovirus. Therefore, this study was carried out to examine virucidal efficacy of the disinfectant tablet against iridovirus.

\section{Materials and Methods}

\section{Disinfectant}

The active ingredients for Easy-Wash ${ }^{\circledR}$, the tested disinfectant tablet, are calcium hypochlorite $(70 \% \mathrm{w} / \mathrm{w})$. Easy-Wash ${ }^{\circledR}$ was provided by Dae Han New Pharm Co. (Seoul, Korea) The disinfectant tablet was stored in the dark in room temperature and prepared for dilution on the day of evaluation. Determination of the antiviral efficacy of the disinfectant was based on Animal, Plant and Fisheries Quarantine and Inspection Agency (APFQIA) Regulation No. 2008-14, Korea ${ }^{11)}$.

\section{Red sea bream iridovirus and culture}

Red sea bream iridovirus (RBIV-Yeosu strain, RBIV) obtained from the College of Fisheries and Ocean Sciences, Chonnam National University (Yeosu, Korea). The RBIV was inoculated in fat head minnow (FHM) cell line and cultured in Eagle's minimum essential medium (MEM, Gibco, Germany) containing $10 \%$ fetal bovine serum at $25^{\circ} \mathrm{C}$ for $4-$ 5 days. After virus growth, infected cells were frozen and thawed three times followed by centrifugation at $400 \times \mathrm{g}$ for $20 \mathrm{~min}$ to remove cell debris. The initial viral titer was $1.1 \times 10^{5}$ tissue culture infecting dose (TCID) $)_{50} / \mathrm{ml}$. All virus stocks were stored in small aliquots at $27^{\circ} \mathrm{C}$ until used.

\section{Diluents and treatment condition}

Testing was based on virucidal effects of disinfectant diluents in three treatment conditions (distilled water (DW) condition, standard hard water (HW) condition, and organic matter (OM) condition), pathogen control (disinfectant

Table 1. Experimental design for the determination of the virucidal efficacy of an aquatic disinfectant tablet

\begin{tabular}{cccccc}
\hline \multirow{2}{*}{$\begin{array}{c}\text { Treatment } \\
\text { condition* }\end{array}$} & \multicolumn{5}{c}{ Contents according to treatment condition** } \\
\cline { 2 - 6 } & DM & HW & OM & Disinfectant & AIV \\
\hline DW condition & + & - & - & + & + \\
HW condition & - & + & - & + & + \\
OM condition & - & - & + & + & + \\
Bacteria control & - & + & - & - & + \\
DW control & + & - & - & - & + \\
\hline
\end{tabular}

*DW, distilled water; HW, standard hard water; OM, organic matter; AIV, avian influenza virus.

**+, presence; -, absence negative control) and DW control (both disinfectant and pathogen negative control) in Table 1. HW, an ingredient of HW treatment condition, was made by adding anhydrous $\mathrm{CaCl}_{2} 0.305 \mathrm{~g}$ and $\mathrm{MgCl}_{2} \cdot 6 \mathrm{H}_{2} \mathrm{O} 0.139 \mathrm{~g}$ into 1 liter distilled water. Organic suspension, an ingredient of OM treatment condition, is a solution of $1 \%(\mathrm{w} / \mathrm{v})$ fetal bovine serum (FBS, Sigma-Aldrich Korea, Seoul) in HW.

\section{Virus-disinfectant contact reaction}

An aquatic disinfectant tablet (Easy-Wash ${ }^{\circledR}$ ) was diluted 23,000, 25,000, 27,000, 29,000, 31,000, and 33,000 times with DW and HW, and diluted 20,000, 22,000, 24,000, 26,000, 28,000 , and 30,000 times with OM, respectively. After dilution of disinfectant, $2.5 \mathrm{ml}$ of disinfectant diluents was added into each test tube.

One $\mathrm{ml}$ of RBIV stock was diluted with $19 \mathrm{ml} \mathrm{DW}$, HW, and OM, respectively. After dilution of the viral stock, $2.5 \mathrm{ml}$ of the diluents was inserted into each test tube containing disinfectant diluents, and incubated at $4^{\circ} \mathrm{C}$ for $30 \mathrm{~min}$.

\section{Evaluation of Easy-Wash against RBIV}

After virus-disinfectant contact reaction, $2.5 \mathrm{ml}$ of $10 \%$ inactivated fetal bovine serum was added into each test tube to neutralize efficacy of disinfectant at room temperature. The neutralized solutions were diluted $10,10^{2}, 10^{3}, 10^{4}, 10^{5}$, and $10^{6}$ times with MEM medium containing $10 \%$ fetal bovine serum and $50 \mu \mathrm{l}$ of the neutralized solution diluents each was injected into five well of FHM cells cultured 96well plate. After inoculation, the cultures were incubated for 5 days at $37^{\circ} \mathrm{C}$ with relative humidity at $85 \%$. The appearance of viral cytopathic effect (CPE) was checked with a microscope everyday during the incubation period.

The validity of concentration for the disinfectant was estimated from the concentration of the dilution that the viral dose in the cell stock treated with Easy-Wash ${ }^{\circledR}$ was inactivated more than $10^{4}$ tissue culture infectious dose $\left(\mathrm{TCID}_{50}\right)$ compared with positive control. $\mathrm{TCID}_{50}$ was calculated according to the method of Käber ${ }^{12)}$. The validity of concentration for an aquatic disinfectant tablet was independently examined on triplicate and determined the validity of concentration with the median of the results.

\section{Results and Discussion}

Table 2-4 present the results of the efficacy testing of an aquatic disinfectant tablet composed to calcium hypochlorite against RBIV. Table 5 shows the summary of the valid dilution time for an aquatic disinfectant tablet against RBIV. On DW and HW condition, RBIV was inactivated with 25,000 fold dilutions of the disinfectant throughout all experiments. With the investigation of the antiviral effect of 
Antiviral Efficacy of an Aquatic Disinfectant Tablet Composed to Calcium Hypochlorite Against Red Sea Bream Iridovirus 287

Table 2. The validation of an aquatic disinfectant tablet against red sea bream iridovirus: first examination

\begin{tabular}{|c|c|c|c|c|c|c|c|c|c|}
\hline \multirow{2}{*}{$\begin{array}{l}\text { Tratment } \\
\text { condition }^{1)}\end{array}$} & \multirow{2}{*}{ Dilution time } & \multicolumn{6}{|c|}{ Dilution time of neutralization solution (positive/the number of inoculation) } & \multirow{2}{*}{$\mathrm{TCID}_{50}^{2)}$} & \multirow{2}{*}{ Log reduction } \\
\hline & & $10^{-1}$ & $10^{-2}$ & $10^{-3}$ & $10^{-4}$ & $10^{-5}$ & $10^{-6}$ & & \\
\hline \multirow{6}{*}{ DW } & 23,000 & $0 / 5$ & $0 / 5$ & $0 / 5$ & $0 / 5$ & $0 / 5$ & $0 / 5$ & $\leq 0.5$ & $\geq 4.1$ \\
\hline & 25,000 & $0 / 5$ & $0 / 5$ & $0 / 5$ & $0 / 5$ & $0 / 5$ & $0 / 5$ & $\leq 0.5$ & $\geq 4.1$ \\
\hline & 27000 & $4 / 5$ & $2 / 5$ & $1 / 5$ & $0 / 5$ & $0 / 5$ & $0 / 5$ & 0.9 & 3.2 \\
\hline & 29,000 & $5 / 5$ & $2 / 5$ & $1 / 5$ & $0 / 5$ & $0 / 5$ & $0 / 5$ & 1.1 & 3.0 \\
\hline & 31,000 & $5 / 5$ & $4 / 5$ & $2 / 5$ & $1 / 5$ & $0 / 5$ & $0 / 5$ & 1.9 & 2.2 \\
\hline & 33,000 & $5 / 5$ & $5 / 5$ & $4 / 5$ & $1 / 5$ & $0 / 5$ & $0 / 5$ & 2.5 & 1.6 \\
\hline \multirow{6}{*}{ HW } & 23,000 & $0 / 5$ & $0 / 5$ & $0 / 5$ & $0 / 5$ & $0 / 5$ & $0 / 5$ & $\leq 0.5$ & $\geq 4.1$ \\
\hline & 25,000 & $4 / 5$ & $2 / 5$ & $1 / 5$ & $0 / 5$ & $0 / 5$ & $0 / 5$ & 0.9 & 3.2 \\
\hline & 27000 & $5 / 5$ & $2 / 5$ & $1 / 5$ & $0 / 5$ & $0 / 5$ & $0 / 5$ & 1.1 & 3.0 \\
\hline & 29,000 & $5 / 5$ & $4 / 5$ & $2 / 5$ & $1 / 5$ & $0 / 5$ & $0 / 5$ & 1.9 & 2.2 \\
\hline & 31,000 & $5 / 5$ & $5 / 5$ & $3 / 5$ & $1 / 5$ & $0 / 5$ & $0 / 5$ & 2.3 & 1.8 \\
\hline & 33,000 & $5 / 5$ & $5 / 5$ & $4 / 5$ & $1 / 5$ & $0 / 5$ & $0 / 5$ & 2.5 & 1.6 \\
\hline \multirow{6}{*}{$\mathrm{OM}$} & 20,000 & $0 / 5$ & $0 / 5$ & $0 / 5$ & $0 / 5$ & $0 / 5$ & $0 / 5$ & $\leq 0.5$ & $\geq 4.1$ \\
\hline & 22,000 & $0 / 5$ & $0 / 5$ & $0 / 5$ & $0 / 5$ & $0 / 5$ & $0 / 5$ & $\leq 0.5$ & $\geq 4.1$ \\
\hline & 24,000 & $4 / 5$ & $3 / 5$ & $1 / 5$ & $0 / 5$ & $0 / 5$ & $0 / 5$ & 1.1 & 3.0 \\
\hline & 26,000 & $5 / 5$ & $4 / 5$ & $1 / 5$ & $0 / 5$ & $0 / 5$ & $0 / 5$ & 1.5 & 2.6 \\
\hline & 28,000 & $5 / 5$ & $5 / 5$ & $2 / 5$ & $1 / 5$ & $0 / 5$ & $0 / 5$ & 2.1 & 2.0 \\
\hline & 30,000 & $5 / 5$ & $5 / 5$ & $4 / 5$ & $1 / 5$ & $0 / 5$ & $0 / 5$ & 2.5 & 1.6 \\
\hline Positive control & & $5 / 5$ & $5 / 5$ & $5 / 5$ & $5 / 5$ & $3 / 5$ & $0 / 5$ & 4.1 & \\
\hline Negative control & & $5 / 5$ & $5 / 5$ & $5 / 5$ & $5 / 5$ & $2 / 5$ & $0 / 5$ & 3.9 & \\
\hline
\end{tabular}

${ }^{1)} \mathrm{DW}$, distilled water; HW, hard water; OM, organic matter.

${ }^{2)} \mathrm{TCID}_{50}=-\mathrm{L} 1-[\mathrm{L} \times\{\mathrm{S} / 100-0.5\}]$

$\left(\mathrm{L}_{1}\right.$, Log of lowest dilution tested; $\mathrm{L}, \log$ interval between dilutions; $\mathrm{S}$, sum of $\%$ mortality at each dilution)

Table 3. The validation of an aquatic disinfectant tablet against red sea bream iridovirus: second examination

\begin{tabular}{|c|c|c|c|c|c|c|c|c|c|}
\hline \multirow{2}{*}{$\begin{array}{l}\text { Tratment } \\
\text { condition }^{1)}\end{array}$} & \multirow{2}{*}{ Dilution time } & \multicolumn{6}{|c|}{ Dilution time of neutralization solution (positive/the number of inoculation) } & \multirow{2}{*}{$\mathrm{TCID}_{50}{ }^{2)}$} & \multirow{2}{*}{ Log reduction } \\
\hline & & $10^{-1}$ & $10^{-2}$ & $10^{-3}$ & $10^{-4}$ & $10^{-5}$ & $10^{-6}$ & & \\
\hline \multirow{6}{*}{ DW } & 23,000 & $0 / 5$ & $0 / 5$ & $0 / 5$ & $0 / 5$ & $0 / 5$ & $0 / 5$ & $\leq 0.5$ & $\geq 4.3$ \\
\hline & 25,000 & $0 / 5$ & $0 / 5$ & $0 / 5$ & $0 / 5$ & $0 / 5$ & $0 / 5$ & $\leq 0.5$ & $\geq 4.3$ \\
\hline & 27000 & $4 / 5$ & $2 / 5$ & $0 / 5$ & $0 / 5$ & $0 / 5$ & $0 / 5$ & 0.7 & 3.6 \\
\hline & 29,000 & $4 / 5$ & $2 / 5$ & $1 / 5$ & $0 / 5$ & $0 / 5$ & $0 / 5$ & 0.9 & 3.4 \\
\hline & 31,000 & $5 / 5$ & $2 / 5$ & $1 / 5$ & $0 / 5$ & $0 / 5$ & $0 / 5$ & 1.1 & 3.2 \\
\hline & 33,000 & $5 / 5$ & $4 / 5$ & $2 / 5$ & $1 / 5$ & $0 / 5$ & $0 / 5$ & 1.9 & 2.4 \\
\hline \multirow{6}{*}{ HW } & 23,000 & $0 / 5$ & $0 / 5$ & $0 / 5$ & $0 / 5$ & $0 / 5$ & $0 / 5$ & $\leq 0.5$ & $\geq 4.3$ \\
\hline & 25,000 & $0 / 5$ & $0 / 5$ & $0 / 5$ & $0 / 5$ & $0 / 5$ & $0 / 5$ & $\leq 0.5$ & $\geq 4.3$ \\
\hline & 27000 & $4 / 5$ & $3 / 5$ & $1 / 5$ & $0 / 5$ & $0 / 5$ & $0 / 5$ & 1.1 & 3.2 \\
\hline & 29,000 & $5 / 5$ & $4 / 5$ & $2 / 5$ & $0 / 5$ & $0 / 5$ & $0 / 5$ & 1.7 & 2.6 \\
\hline & 31,000 & $5 / 5$ & $5 / 5$ & $3 / 5$ & $1 / 5$ & $0 / 5$ & $0 / 5$ & 2.3 & 2.0 \\
\hline & 33,000 & $5 / 5$ & $5 / 5$ & $4 / 5$ & $1 / 5$ & $0 / 5$ & $0 / 5$ & 2.5 & 1.8 \\
\hline \multirow{6}{*}{$\mathrm{OM}$} & 20,000 & $0 / 5$ & $0 / 5$ & $0 / 5$ & $0 / 5$ & $0 / 5$ & $0 / 5$ & $\leq 0.5$ & $\geq 4.3$ \\
\hline & 22,000 & $4 / 5$ & $2 / 5$ & $1 / 5$ & $0 / 5$ & $0 / 5$ & $0 / 5$ & 0.9 & 3.4 \\
\hline & 24,000 & $4 / 5$ & $3 / 5$ & $1 / 5$ & $0 / 5$ & $0 / 5$ & $0 / 5$ & 1.1 & 3.2 \\
\hline & 26,000 & $5 / 5$ & $4 / 5$ & $2 / 5$ & $0 / 5$ & $0 / 5$ & $0 / 5$ & 1.7 & 2.6 \\
\hline & 28,000 & $5 / 5$ & $4 / 5$ & $2 / 5$ & $1 / 5$ & $0 / 5$ & $0 / 5$ & 1.9 & 2.4 \\
\hline & 30,000 & $5 / 5$ & $5 / 5$ & $4 / 5$ & $1 / 5$ & $0 / 5$ & $0 / 5$ & 2.5 & 1.8 \\
\hline Positive control & & $5 / 5$ & $5 / 5$ & $5 / 5$ & $5 / 5$ & $4 / 5$ & $0 / 5$ & 4.3 & \\
\hline Negative control & & $5 / 5$ & $5 / 5$ & $5 / 5$ & $5 / 5$ & $3 / 5$ & $0 / 5$ & 4.1 & \\
\hline
\end{tabular}

${ }^{10} \mathrm{DW}$, distilled water; HW, hard water; OM, organic matter.

${ }^{2)} \mathrm{TCID}_{50}=-\mathrm{L} 1-[\mathrm{L} \times\{\mathrm{S} / 100-0.5\}]$

$\left(\mathrm{L}_{1}, \mathrm{Log}\right.$ of lowest dilution tested; $\mathrm{L}, \log$ interval between dilutions; $\mathrm{S}$, sum of $\%$ mortality at each dilution) 
Table 4. The validation of an aquatic disinfectant tablet against red sea bream iridovirus: third examination

\begin{tabular}{|c|c|c|c|c|c|c|c|c|c|}
\hline \multirow{2}{*}{$\begin{array}{l}\text { Tratment } \\
\text { condition }^{1)}\end{array}$} & \multirow{2}{*}{ Dilution time } & \multicolumn{6}{|c|}{ Dilution time of neutralization solution (positive/the number of inoculation) } & \multirow{2}{*}{$\mathrm{TCID}_{50}{ }^{2)}$} & \multirow{2}{*}{ Log reduction } \\
\hline & & $10^{-1}$ & $10^{-2}$ & $10^{-3}$ & $10^{-4}$ & $10^{-5}$ & $10^{-6}$ & & \\
\hline \multirow{6}{*}{ DW } & 23,000 & $0 / 5$ & $0 / 5$ & $0 / 5$ & $0 / 5$ & $0 / 5$ & $0 / 5$ & $\leq 0.5$ & $\geq 4.1$ \\
\hline & 25,000 & $4 / 5$ & $2 / 5$ & $1 / 5$ & $0 / 5$ & $0 / 5$ & $0 / 5$ & 0.9 & 3.2 \\
\hline & 27000 & $5 / 5$ & $3 / 5$ & $1 / 5$ & $0 / 5$ & $0 / 5$ & $0 / 5$ & 1.3 & 2.8 \\
\hline & 29,000 & $5 / 5$ & $4 / 5$ & $1 / 5$ & $0 / 5$ & $0 / 5$ & $0 / 5$ & 1.5 & 2.6 \\
\hline & 31,000 & $5 / 5$ & $5 / 5$ & $2 / 5$ & $0 / 5$ & $0 / 5$ & $0 / 5$ & 1.9 & 2.2 \\
\hline & 33,000 & $5 / 5$ & $5 / 5$ & $3 / 5$ & $1 / 5$ & $0 / 5$ & $0 / 5$ & 2.3 & 1.8 \\
\hline \multirow{6}{*}{ HW } & 23,000 & $0 / 5$ & $0 / 5$ & $0 / 5$ & $0 / 5$ & $0 / 5$ & $0 / 5$ & $\leq 0.5$ & $\geq 4.1$ \\
\hline & 25,000 & $0 / 5$ & $0 / 5$ & $0 / 5$ & $0 / 5$ & $0 / 5$ & $0 / 5$ & $\leq 0.5$ & $\geq 4.1$ \\
\hline & 27000 & $4 / 5$ & $2 / 5$ & $1 / 5$ & $0 / 5$ & $0 / 5$ & $0 / 5$ & 0.9 & 3.2 \\
\hline & 29,000 & $4 / 5$ & $3 / 5$ & $1 / 5$ & $0 / 5$ & $0 / 5$ & $0 / 5$ & 1.1 & 3.0 \\
\hline & 31,000 & $5 / 5$ & $4 / 5$ & $3 / 5$ & $1 / 5$ & $0 / 5$ & $0 / 5$ & 2.1 & 2.0 \\
\hline & 33,000 & $5 / 5$ & $5 / 5$ & $4 / 5$ & $1 / 5$ & $0 / 5$ & $0 / 5$ & 2.5 & 1.6 \\
\hline \multirow{6}{*}{$\mathrm{OM}$} & 20,000 & $0 / 5$ & $0 / 5$ & $0 / 5$ & $0 / 5$ & $0 / 5$ & $0 / 5$ & $\leq 0.5$ & $\geq 4.1$ \\
\hline & 22,000 & $0 / 5$ & $0 / 5$ & $0 / 5$ & $0 / 5$ & $0 / 5$ & $0 / 5$ & $\leq 0.5$ & $\geq 4.1$ \\
\hline & 24,000 & $4 / 5$ & $3 / 5$ & $1 / 5$ & $0 / 5$ & $0 / 5$ & $0 / 5$ & 1.1 & 3.0 \\
\hline & 26,000 & $5 / 5$ & $4 / 5$ & $1 / 5$ & $0 / 5$ & $0 / 5$ & $0 / 5$ & 1.5 & 2.6 \\
\hline & 28,000 & $5 / 5$ & $5 / 5$ & $3 / 5$ & $1 / 5$ & $0 / 5$ & $0 / 5$ & 2.3 & 1.8 \\
\hline & 30,000 & $5 / 5$ & $5 / 5$ & $4 / 5$ & $2 / 5$ & $0 / 5$ & $0 / 5$ & 2.7 & 1.4 \\
\hline Positive control & & $5 / 5$ & $5 / 5$ & $5 / 5$ & $5 / 5$ & $3 / 5$ & $0 / 5$ & 4.1 & \\
\hline Negative control & & $5 / 5$ & $5 / 5$ & $5 / 5$ & $5 / 5$ & $2 / 5$ & $0 / 5$ & 3.9 & \\
\hline
\end{tabular}

${ }^{10} \mathrm{DW}$, distilled water; HW, hard water; OM, organic matter.

${ }^{2)} \mathrm{TCID}_{50}=-\mathrm{L} 1-[\mathrm{L} \times\{\mathrm{S} / 100-0.5\}]$

$\left(\mathrm{L}_{1}, \mathrm{Log}\right.$ of lowest dilution tested; $\mathrm{L}, \log$ interval between dilutions; $\mathrm{S}$, sum of $\%$ mortality at each dilution)

Table 5. The summary of the valid dilution time for an aquatic disinfectant tablet against red sea bream iridovirus

\begin{tabular}{ccccc}
\hline \multirow{2}{*}{$\begin{array}{c}\text { Tretment } \\
\text { condition }\end{array}$} & \multicolumn{3}{c}{ Experiment } & \multirow{2}{*}{ Median } \\
\cline { 2 - 4 } & first & second & third & \\
\hline DW & $1 / 25,000$ & $1 / 25,000$ & $1 / 23,000$ & $1 / 25,000$ \\
HW & $1 / 23,000$ & $1 / 25,000$ & $1 / 25,000$ & $1 / 25,000$ \\
OM & $1 / 22,000$ & $1 / 20,000$ & $1 / 22,000$ & $1 / 22,000$ \\
$\begin{array}{c}\text { Positive } \\
\text { control }\end{array}$ & 4.1 & 4.3 & 4.1 & \\
$\begin{array}{c}\text { Negative } \\
\text { control }\end{array}$ & 3.9 & 4.1 & 3.9 & \\
\hline
\end{tabular}

${ }^{10} \mathrm{DW}$, distilled water; HW, hard water; OM, organic matter.

the disinfectant on OM condition, RBIV was inactivated on 22,000 fold dilutions.

As organic material interferes with efficacy by either inactivating the disinfectant or blocking it from surface contact, the virucidal activity of an aquatic disinfectant tablet on the OM condition lowered efficacy against RBIV compared with DM or HW conditions.

Yamashita et al. (2005) reported that fish viruses including iridovirus were sensitive to more than $0.5 \%$ formalin ${ }^{13)}$. In addition, Fan et al. (2012) reported that DNA-containing turbot reddish body iridovirus was completely inactivated by
$0.1 \%$ formalin within $48 \mathrm{~h}$ at $37^{\circ} \mathrm{C}^{14)}$. Due to the economic and reliable disinfect, formalin has been widely used as a disinfectant for decontamination of cultured marine fish and its facilities. However, formalin had to be replaced in many countries including France because of its potential carcinogenic effects on human health and environmental concerns ${ }^{15)}$.

In the present study, the disinfectant efficacy of an aquatic disinfectant tablet against RBIV showed higher than formalin tested by Yamashita et al. ${ }^{13)}$ and Fan et al. ${ }^{14)}$.

In this study, disinfectant efficacy of an aquatic disinfectant tablet has a limitation that the results are based on in vitro test. Organic material in suspension (OM condition) could not represent all possible parameters of RBIV contaminated environments.

As the efficacy of an aquatic disinfectant tablet against RBIV was investigated in vitro, a controlled field trial is required to determine whether the use of an aquatic disinfectant tablet will be able to reduce RBIV in cultured marine fish farm.

\section{Acknowledgements}

This work was financially supported by Dae Han New Pharm Co., LTD. (Seoul, Korea). 


\section{References}

1. Plumb, J.A., Grizzle, J.M., Young, H.E. and Noyes, A.D.: An iridovirus isolated from wild largemouth bass. J. Aquat. Anim. Health 8, 265-270 (1996).

2. Park, S.J., Seo, H.J., Son, J.H., Kim, H.J., Kim, Y.I., Kim, K.H., Nam, Y.K. and Kim, S.K.: Development of DNA vaccine against red sea bream iridovirus. J. Microbiol. Biotechnol. 15, 873-879 (2005).

3. Oshima, S., Hata, J., Hiraswa, N., Ohtaka, T., Hirono, I., Aoki, T., Yamashital, S.: Rapid diagnosis of red sea bream iridovirus infection using the polymerase chain reactionol. Dis. Aquat. Org. 32, 87-90 (1998).

4. Williams, T., Barbosa-Solomieu, V. and Chinchar, V.G.: A decade of advances in iridovirus research. Adv. Virus Res. 65, 173-248 (2005).

5. Inouye, K., Yamano, K., Maeno, Y., Nakajima, K., Matsuoka, M., Wada, Y. and Sorimachi, M.: Iridovirus infection of cultured red sea bream, Pagrus major. Fish Pathol. 27, 19-27 (1992).

6. Do, J.W., Cha, S.J., Kim, J.S., An, E.J., Lee, N.S., Choi, H.J., Lee, C.H., Park, M.S., Kim, J.W., Kim, Y.C. and Park, J.W.: Phylogenetic analysis of the major capsid protein gene of iridovirus isolates from cultured flounders Paralichthys olivaceus in Korea. Dis. Aquat. Org. 64, 193-200 (2005).

7. Gibson-Kueh, S., Netto, P., Ngoh-Lim, G.H., Chang, S.F., Ho, L.L., Qin, Q.W., Chua, F.H.C., Ng, M.L. and Ferguson, H.W.:
The pathlogy of systemic iridovirus disease in fish. J. Comp. Pathol. 129, 111-119 (2003).

8. Kasai, H., Yoshimizu, M. AND Ezura, Y.: Disinfection of water for aquaculture. Fish Sci. 68, 821-824 (2002).

9. The Center for Food Security and Public Health: Epizootic hematopoietic necrosis. Iowa State University Press, Ames, pp. 1-4 (2007).

10. Yoshmizu, M., Yoshinaka, T., Hatori, S. and Hisae Kasal, H.: Survivability of fish pathogenic viruses in environmental water, and inactivation of fish viruses. Bull. Fish Res. Agen. 2, 47-54 (2005).

11. Animal, Plant and Fisheries Quarantine and Inspection Agency (APFQIA): The guideline of the efficacy test for disinfectants. APFQIA Regulation No. 2008-14, APFQIA, Seoul (2008).

12. Kärber, G.: Beitrag zur kollektiven Behandlung pharmakologischer Reihenversuche. Arch. Exp. Pathol. Pharmakol., 162, 480-487 (1931).

13. Yamashita, H., Fujita, Y., Kawakami, H., and Nakai, T.: The efficacy of inactivated virus vaccine against viral nervous necrosis (NNV). Fish Pathol. 40, 15-21 (2005).

14. Fan, T., Hu, X., Wang, L., Geng, X., Jiang, G., Yang, X. and Yu, M.: Development of an inactivated iridovirus vaccine against turbot viral reddish body syndrome. J. Ocean Univ. Chana, 11, 65-69 (2011).

15. Epizone: Workshop - Formaldehyde replacement, Lelystad, 11-12 January 2011. Central Veterinary Institute of Wageningen UR, Lelystad, pp. 4-8 (2011). 